PROCEEDINGS OF THE

AMERICAN MATHEMATICAL SOCIETY

Volume 130, Number 5, Pages 1279-1283

S 0002-9939(01)06197-4

Article electronically published on October 12, 2001

\title{
COMMUTATOR INEQUALITIES ASSOCIATED WITH THE POLAR DECOMPOSITION
}

\author{
FUAD KITTANEH
}

(Communicated by Joseph A. Ball)

\begin{abstract}
Let $A=U P$ be a polar decomposition of an $n \times n$ complex matrix $A$. Then for every unitarily invariant norm $\|\mid \cdot\| \|$, it is shown that

$$
|||| U P-\left.P U\right|^{2}||\left|\leq\left\|A^{*} A-A A^{*}|||\leq\|U P+P U\|\|U P-P U \mid\|,\right.\right.
$$

where $\|\cdot\|$ denotes the operator norm. This is a quantitative version of the wellknown result that $A$ is normal if and only if $U P=P U$. Related inequalities involving self-commutators are also obtained.
\end{abstract}

\section{INTRODUCTION}

Let $M_{n}(\mathbb{C})$ be the space of all $n \times n$ complex matrices. For $A \in M_{n}(\mathbb{C})$ there exists a unitary matrix $U$ and a positive semidefinite matrix $P$ such that

$$
A=U P .
$$

The decomposition (1) is called a polar decomposition of $A$. In this decomposition the positive semidefinite part $P$ is unique and $P=|A|=\left(A^{*} A\right)^{1 / 2}$. The unitary part $U$ is unique if $A$ is invertible. It is obvious that $A^{*} A=P^{2}$ and $A A^{*}=$ $U P^{2} U^{*}=\left(U P U^{*}\right)^{2}$. Thus, it follows from the spectral theorem that $A$ is normal (i.e., $A^{*} A=A A^{*}$ ) if and only if $U P=P U$. Equivalently, the self-commutator $A^{*} A-A A^{*}$ is zero if and only if the commutator $U P-P U$ is zero (see [1, p. 6], [6, p. 170], or [8, p. 414]).

The main purpose of this paper is to establish norm inequalities for self-commutators of matrices. Though we confine our discussion to matrices regarded as operators on a finite-dimensional Hilbert space, by slight modifications the inequalities we obtain here can be extended to operators on an infinite-dimensional Hilbert space.

In Section 2 we present norm inequalities comparing the self-commutator $A^{*} A-$ $A A^{*}$ and the commutator $U P-P U$. It will be evident from these inequalities that a matrix $A$ is normal if and only if the factors $U$ and $P$ in the polar decomposition of $A$ commute.

It has been shown by Fong [5] that if $A \in M_{n}(\mathbb{C})$, then

$$
\left\|A^{*} A-A A^{*}\right\| \leq\|A\|^{2},
$$

Received by the editors December 14, 1999 and, in revised form, November 1, 2000.

2000 Mathematics Subject Classification. Primary 15A23, 15A57, 15A60, 47A30, 47B47.

Key words and phrases. Commutator, polar decomposition, positive semidefinite matrix, unitarily invariant norm. 
where $\|\cdot\|$ denotes the operator (or the spectral) norm. A different proof of inequality (2) with an extension to the Schatten $p$-norms can be found in [3].

In Section 3 we give a norm inequality that complements (2). In fact, it will be shown that

$$
\|A\|^{2}-\left\|A^{2}\right\| \leq\left\|A^{*} A-A A^{*}\right\| .
$$

The example $A=\left[\begin{array}{ll}0 & 1 \\ 0 & 0\end{array}\right]$ shows that inequalities (2) and (3) are sharp. It follows immediately from inequality (3) that if $A$ is normal, then $\left\|A^{2}\right\|=\|A\|^{2}$, which is a well-known fact (see, e.g., [6, p. 178]).

\section{Inequalities associated With the POLAR DECOMPosition}

In this section we present quantitative versions of the characterization that a matrix $A$ is normal if and only if the factors $U$ and $P$ in the polar decomposition of $A$ commute. To achieve our goal, we need several lemmas, in which and in the sequel ||$|\cdot|||$ designates any unitarily invariant norm.

Lemma 1. If $X, Y \in M_{n}(\mathbb{C})$, then

$$
\left\|\left|X^{2}-Y^{2}\right|\right\| \leq\|X+Y\||\|X-Y \mid\| .
$$

To prove (4), one needs to use the identity

$$
X^{2}-Y^{2}=\frac{1}{2}(X+Y)(X-Y)+\frac{1}{2}(X-Y)(X+Y),
$$

the triangle inequality, and the fact that for all $B, C \in M_{n}(\mathbb{C}),|||B C| \|$ is dominated by both $\|B\|\||| C \mid\|$ and $\|C\|\|\| B \| \mid$ (see, e.g., [1, p. 94]).

Lemma 2. If $X, Y \in M_{n}(\mathbb{C})$ are positive semidefinite, then

$$
\|||(X-Y)^{2}|||\leq||| X^{2}-Y^{2}|| \mid .
$$

For a proof of a more general form of Lemma 2, the reader is referred to [1] p. 294] and references therein.

The following related inequality can be found in [1. p. 318] (see also [2, Proposition 6]).

Lemma 3. If $X, Y \in M_{n}(\mathbb{C})$ are positive semidefinite such that $X+Y \geq$ aI for some $a \geq 0$, then

$$
a|| X-Y|\|| \leq|| X^{2}-Y^{2}|| \mid
$$

For the particularly important Hilbert-Schmidt (or Frobenius) norm $\|\cdot\|_{2}$, we have the following refined version of Lemma 2 ,

Lemma 4. If $X, Y \in M_{n}(\mathbb{C})$ are positive semidefinite, then

$$
\|X Y-Y X\|_{2}^{2}+\left\|(X-Y)^{2}\right\|_{2}^{2} \leq\left\|X^{2}-Y^{2}\right\|_{2}^{2} .
$$

Proof. It is known that if $X, Y \in M_{n}(\mathbb{C})$ are positive semidefinite, then for all $Z \in M_{n}(\mathbb{C})$, we have

$$
\|X Z-Z Y\|_{2} \leq\|X Z+Z Y\|_{2} .
$$

Indeed, inequality (9) follows from the identity

$$
\|X Z+Y Z\|_{2}^{2}=\|X Z-Y Z\|_{2}^{2}+4\left\|X^{1 / 2} Z Y^{1 / 2}\right\|_{2}^{2},
$$


which can be easily verified by using the facts that $\|B\|_{2}^{2}=\operatorname{tr} B^{*} B$ and $\operatorname{tr} B C=$ $\operatorname{tr} C B$ for all $B, C \in M_{n}(\mathbb{C})$, where tr stands for the trace functional (see [9]). Letting $Z=X-Y$ in (9), we conclude that

$$
\left\|X^{2}-2 X Y+Y^{2}\right\|_{2} \leq\left\|X^{2}-Y^{2}\right\|_{2}
$$

Now observe that

$$
\begin{aligned}
& \operatorname{Re}\left(X^{2}-2 X Y+Y^{2}\right)=(X-Y)^{2}, \\
& \operatorname{Im}\left(X^{2}-2 X Y+Y^{2}\right)=i(X Y-Y X),
\end{aligned}
$$

where, for any $B \in M_{n}(\mathbb{C}), \operatorname{Re} B$ and $\operatorname{Im} B$ are the matrices $\frac{1}{2}\left(B+B^{*}\right)$ and $\frac{1}{2 i}\left(B-B^{*}\right)$, respectively. Since $\|B\|_{2}^{2}=\|\operatorname{Re} B\|_{2}^{2}+\|\operatorname{Im} B\|_{2}^{2}$, inequality (8) follows from (II). This completes the proof.

For inequalities related to inequalities (8), (91), and (11), we refer to [4], [7], and 10].

Now we utilize the previous lemmas to prove our commutator inequalities that are associated with the polar decomposition.

Theorem 1. Let $A \in M_{n}(\mathbb{C})$ with a polar decomposition $A=U P$. Then

$$
\text { || |UP }-\left.P U\right|^{2}\left|\left\|\leq\left|\left\|A^{*} A-A A^{*} \mid\right\| \leq\|U P+P U\|\|U P-P U\| .\right.\right.\right.
$$

In particular, for the operator norm, we have

$$
\|U P-P U\|^{2} \leq\left\|A^{*} A-A A^{*}\right\| \leq\|U P+P U\|\|U P-P U\| .
$$

$$
2 s_{n}(A)|\|U P-P U\|| \leq\left\|A^{*} A-A A^{*}\left|\left\|\leq 2 s_{1}(A)\right\|\right| U P-P U\right\|,
$$

where $s_{1}(A)$ and $s_{n}(A)$ are the largest and smallest singular values of $A$, respectively,

$$
\begin{aligned}
& \left\|P U P U^{*}-U P U^{*} P\right\|_{2}^{2}+\left\||U P-P U|^{2}\right\|_{2}^{2} \\
& \quad \leq\left\|A^{*} A-A A^{*}\right\|_{2}^{2} \leq\|U P+P U\|^{2}\|U P-P U\|_{2}^{2} .
\end{aligned}
$$

Proof. We have $P^{2}-\left(U P U^{*}\right)^{2}=A^{*} A-A A^{*}$. So, applying Lemmas 1 and 2 to the positive semidefinite matrices $P$ and $U P U^{*}$, we obtain

$$
\left\|\left|\left(P-U P U^{*}\right)^{2}\right|\right\| \leq\left\|A^{*} A-A A^{*}\right\|\left|\leq\left\|P+U P U^{*}\right\|\left\|P-U P U^{*} \mid\right\| .\right.
$$

Using the unitary invariance of these norms and the fact that $\left.|||| B\right|^{2}|||=|||\left|B^{*}\right|^{2}|| \mid$ for every $B \in M_{n}(\mathbb{C})$, we have

$$
\begin{aligned}
& ||\left|\left(P-U P U^{*}\right)^{2}\right|||=|||| P-\left.U P U^{*}\right|^{2}|||=|||\left|U\left(U^{*} P-P U^{*}\right)\right|^{2}|| \mid \\
& =|||| U^{*} P-\left.P U^{*}\right|^{2}|||=||||U P-P U|^{2}|| \mid, \\
& || P-U P U^{*}\left|\left\||=|||(P U-U P) U^{*}|||=||| U P-P U \mid\right\|,\right.
\end{aligned}
$$

and

$$
\left\|P+U P U^{*}\right\|=\left\|(P U+U P) U^{*}\right\|=\|U P+P U\| .
$$

These relations, together with (16), yield inequalities (12), (13), and the second inequality in (15). The first inequality in (15), which is a refinement of that in (12) for the Hilbert-Schmidt norm, can be obtained from Lemma 4 by a similar argument. The first inequality in (14) follows by invoking Lemma 3, in view of the fact that $P \geq s_{n}(A) I$ and $U P U^{*} \geq s_{n}(A) I$. Finally, the second inequality in (14) 
follows from that in (12), together with the triangle inequality and the fact that $\|P\|=s_{1}(A)$.

\section{A PROOF OF INEQUALITY (3)}

This section is devoted to the proof of inequality (3). To accomplish this, we need some lemmas, in which we regard the direct sum $B \oplus C$ as the block diagonal matrix $\left[\begin{array}{cc}B & 0 \\ 0 & C\end{array}\right]$. Though inequality (3) is solely connected with the operator norm, we formulate these lemmas in the general context of unitarily invariant norms.

Recall that

$$
\|\| B \oplus C \mid \|=\Phi\left(s_{1}(B), s_{1}(C), \ldots, s_{n}(B), s_{n}(C)\right),
$$

where $\Phi$ is the symmetric gauge function associated with $\||\cdot|\|$, and $s_{1}(B) \geq \cdots \geq$ $s_{n}(B)$ and $s_{1}(C) \geq \cdots \geq s_{n}(C)$ are the singular values of $B$ and $C$, respectively. Since the operator norm of a matrix is the same as its largest singular value, it follows from (17) that

$$
\|B \oplus C\|=\max (\|B\|,\|C\|) .
$$

The following lemma (see [11, Corollaries 1 and 2]) plays a central role in our analysis. Related results, including an improvement for the usual operator norm, can be found in [12].

Lemma 5. If $X, Y \in M_{n}(\mathbb{C})$ are positive semidefinite, then

$$
\||(X+Y) \oplus 0||| \leq|||X \oplus Y|\|+\left\|\left|X^{1 / 2} Y^{1 / 2} \oplus X^{1 / 2} Y^{1 / 2}\right|\right\| .
$$

In particular, for the operator norm, we have

$$
\|X+Y\| \leq \max (\|X\|,\|Y\|)+\left\|X^{1 / 2} Y^{1 / 2}\right\| .
$$

Lemma 6. If $X, Y \in M_{n}(\mathbb{C})$ are positive semidefinite, then

$$
2 s_{j}\left(X^{1 / 2} Y^{1 / 2}\right) \leq s_{j}(X+Y) \text { for } j=1, \ldots, n,
$$

and consequently,

$$
2||\left|X^{1 / 2} Y^{1 / 2}\right||| \leq|| X+Y \| \mid
$$

Inequality (22), which is closely related to (19), is an arithmetic-geometric mean inequality for matrices (see [1, p. 262], [3], and 4]). Though our proof of inequality (3) does not depend on Lemma 6, it is presented here to give a lower bound estimate in the following lemma.

Lemma 7. If $A \in B(H)$, then

$$
2||\left|A^{2} \oplus 0\right||| \leq||\left|\left(A^{*} A+A A^{*}\right) \oplus 0\right||| \leq||\left|A^{*} A \oplus A^{*} A\right|||+||\left|A^{2} \oplus A^{2}\right||| .
$$

In particular, for the operator norm, we have

$$
2\left\|A^{2}\right\| \leq\left\|A^{*} A+A A^{*}\right\| \leq\|A\|^{2}+\left\|A^{2}\right\| .
$$

Proof. If $A=U P$ is a polar decomposition of $A$, then by the unitary invariance of the singular values and Lemma 6, applied to the positive semidefinite matrices $P$ and $U P U^{*}$, we have

$$
\begin{aligned}
2 s_{j}\left(A^{2}\right) & =2 s_{j}(U P U P)=2 s_{j}(P U P)=2 s_{j}\left(P U P U^{*}\right) \\
& \leq s_{j}\left(P^{2}+\left(U P U^{*}\right)^{2}\right)=s_{j}\left(A^{*} A+A A^{*}\right) \text { for } j=1, \ldots, n .
\end{aligned}
$$


This yields the first inequalities in (23) and (24), since the unitarily invariant norms are increasing with respect to the singular values.

The second inequality in (23) follows from Lemma 5, applied to the positive semidefinite matrices $A^{*} A$ and $A A^{*}$, the identity (17), and the facts that $s_{j}\left(A^{*} A\right)=$ $s_{j}\left(A A^{*}\right)$ and $s_{j}\left(|A|\left|A^{*}\right|\right)=s_{j}\left(P U P U^{*}\right)=s_{j}(P U P)=s_{j}\left(A^{2}\right)$ for $j=1, \ldots, n$. The second inequality in (24) follows from that in (23), the identity (18), and the fact that $\left\|A^{*} A\right\|=\|A\|^{2}$.

Now we are in a position to prove inequality (3), which, when combined with inequality (2), gives sharp estimates for the operator norm of self-commutators. Recall that if $A \in M_{n}(\mathbb{C})$, then $\left\|A^{2}\right\| \leq\|A\|^{2}$.

Theorem 2. If $A \in M_{n}(\mathbb{C})$, then

$$
\|A\|^{2}-\left\|A^{2}\right\| \leq\left\|A^{*} A-A A^{*}\right\| \leq\|A\|^{2} .
$$

Proof. In view of inequality (2), we need only prove the first inequality in (25). Using the triangle inequality and the second inequality in (24), we have

$$
\begin{aligned}
\left\|A^{*} A-A A^{*}\right\| & =\left\|A^{*} A+A A^{*}-2 A A^{*}\right\| \\
& \geq 2\left\|A A^{*}\right\|-\left\|A^{*} A+A A^{*}\right\| \\
& \geq 2\|A\|^{2}-\left(\|A\|^{2}+\left\|A^{2}\right\|\right) \\
& =\|A\|^{2}-\left\|A^{2}\right\|,
\end{aligned}
$$

as required.

\section{REFERENCES}

1. R. Bhatia, Matrix Analysis, Springer-Verlag, New York, 1997. MR 98i:15003

2. R. Bhatia and F. Kittaneh, On some perturbation inequalities for operators, Linear Algebra Appl. 106 (1988), 271-279. MR 90f:47007

3. R. Bhatia and F. Kittaneh, On the singular values of a product of operators, SIAM J. Matrix Anal. Appl. 11 (1990), 272-277. MR 90m:47033

4. R. Bhatia and F. Kittaneh, Notes on matrix arithmetic-geometric mean inequalities, Linear Algebra Appl. 308 (2000), 203-211. MR 2001a:15023

5. C. K. Fong, Norm estimates related to self-commutators, Linear Algebra Appl. 74 (1986), 151-156. MR 87c:15047

6. P. R. Halmos, Finite-Dimensional Vector Spaces, Springer-Verlag, New York, 1974. MR 53:13258

7. O. Hirzallah and F. Kittaneh, Matrix Young inequalities for the Hilbert-Schmidt norm, Linear Algebra Appl. 308 (2000), 77-84. MR 2001b:15027

8. R. A. Horn and C. R. Johnson, Matrix Analysis, Cambridge University Press, Cambridge, 1985. MR 87e:15001

9. F. Kittaneh, A note on the arithmetic-geometric mean inequality for matrices, Linear Algebra Appl. 171 (1992), 1-8. MR 93c:15018

10. F. Kittaneh, On some operator inequalities, Linear Algebra Appl. 208/209 (1994), 19-28. MR 95d:47015

11. F. Kittaneh, Norm inequalities for certain operator sums, J. Funct. Anal. 143 (1997), 337348. MR 97k:47005

12. F. Kittaneh, Norm inequalities for sums of positive operators, J. Operator Theory, to appear.

Department of Mathematics, University of Jordan, Amman, Jordan

E-mail address: fkitt@ju.edu.jo 